\title{
Early life sleep disruption drives lasting sex-specific changes in behavior in genetically vulnerable Shank3 heterozygous autism model mice
}

Julia S. Lord ${ }^{1}$, Sean M. Gay ${ }^{1}$, Kathryn M. Harper,2,3, Viktoriya D. Nikolova²,3, Kirsten M. Smith, Sheryl S. Moy ${ }^{\star 2,3}$, Graham H. Diering ${ }^{\star * 1,3}$

Affiliations

1. Department of Cell Biology and Physiology and the Neuroscience Center, University of North Carolina at Chapel Hill, Chapel Hill, NC, USA 27599

2. Department of Psychiatry

3. Carolina Institute for Developmental Disabilities

*Correspondence: sheryl_moy@med.unc.edu

${ }^{* *}$ Correspondence: graham_diering@med.unc.edu

\begin{abstract}
Sleep disruption is a common comorbidity in patients with autism spectrum disorder (ASD), a condition diagnosed with a striking male bias of $\sim 4: 1$. It is unclear how sleep disruption contributes to ASD susceptibility, and the sex biased vulnerability. We examined sleep behavior and the effects of early life sleep disruption (ELSD) in developing mice bearing C-terminal truncation $(\Delta \mathrm{C})$ in $\mathrm{ASD}$ risk gene Shank3. Male and female Shank $3^{\Delta \mathrm{C} / \Delta \mathrm{C}}$ homozygotes showed clear sleep disruption early in postnatal life, compared to Shank ${ }^{\mathrm{WT} / \Delta \mathrm{C}}$ heterozygotes and wildtype littermates, suggesting that sleep disruption may be an early symptom in the expression of ASD. We find that ELSD interacts with genetic vulnerability in Shank $3^{\mathrm{WT} / \Delta \mathrm{C}}$ heterozygotes to drive lasting and sex-specific changes in behavior. Our results clearly show that sleep disruption during sensitive periods of postnatal development is causative of lasting changes in behavior in genetically vulnerable individuals, but in a striking sex-specific manner.
\end{abstract}

\section{Introduction}

Sleep is a conserved and essential physiological process that supports neuronal circuit development and lifelong brain health. In humans and other mammals, sleep undergoes dramatic changes in total amount and composition during the period of early development ${ }^{1-4}$, suggesting that early life sleep is intimately connected to brain development. Up to $80 \%$ of individuals with autism spectrum disorder (ASD) suffer from impaired sleep ${ }^{5-7}$. It is unclear whether sleep disruption, particularly during development, directly contributes to altered behavior and brain function in ASD. Recent studies using rodent or fruit fly models showed that early life sleep disruption results in altered cognitive and social behaviors in adults, with a male bias, strongly suggesting that sleep disruption during development can cause lasting ASD relevant phenotypes ${ }^{8,9}$.

SHANK3 is a high confidence autism risk gene ${ }^{10}$ that encodes a prominent excitatory postsynaptic scaffold protein. Heterozygous mutation or chromosomal loss of SHANK3 is causative of Phelan-McDermid syndrome, a severe neurodevelopmental condition with associated autism and sleep disruption ${ }^{11,12}$. ASD model mice with homozygous mutations in Shank3 exhibit a variety of ASD-relevant phenotypes, while the more clinically relevant Shank3 heterozygotes show no or mild phenotypes ${ }^{13-17}$, often leading to the use of Shank3 homozygous mice as a 
preferred research model. A recent study has documented that adult male mice with homozygous C-terminal truncation of Shank3 (Shank $3^{\Delta \mathrm{C} / \Delta \mathrm{C}}$ ) show clear sleep disruption, reminiscent of patients with Phelan-McDermid syndrome ${ }^{18}$. We expand this finding, demonstrating that sleep disruption is seen in developing Shank $3^{\Delta \mathrm{C} / \Delta \mathrm{C}}$ homozygous mutants of both sexes, but not in Shank $3^{\mathrm{WT} / \Delta \mathrm{C}}$ heterozygotes. Because the Shank ${ }^{\mathrm{WT} / \Delta \mathrm{C}}$ heterozygotes do not intrinsically develop overt sleep disruption, these mice are an ideal model to examine the causal role of developmental sleep disruption in lasting ASD relevant behavior phenotypes. Our results show that early life sleep disruption, but not post-adolescent sleep disruption, can cause lasting and sex-specific changes in behavior in the genetically vulnerable and clinically relevant Shank3 heterozygous mice. Together, our findings suggest sleep disruption is a phenotype that can emerge early in ASD and may be an important factor in lasting changes in brain function and behavior.

\section{Results}

\section{Sleep phenotypes in male and female Shank3 $\Delta$ C ASD model mice}

We first tested whether Shank3 $\Delta$ C ASD model mice exhibited sleep disruption during development. We measured total sleep amount and bout lengths in male and female Shank $3^{\mathrm{WT} / \Delta \mathrm{C}}$ heterozygotes, Shank $3^{\Delta \mathrm{C} / \Delta \mathrm{C}}$ homozygotes, and wild-type (WT) littermates, at two developmental time points: juvenile (P25-P41), and adolescent (P42-P56). Wake and sleep behavior was monitored for an average of 8-10 uninterrupted days using a non-invasive piezoelectric home-cage recording system called PiezoSleep. This system has been well validated using simultaneous EEG/EMG recordings ${ }^{19}$. Average daily total sleep amount and bout lengths were calculated from repeated days of uninterrupted recording for each individual to yield a robust measurement of typical daily sleep behavior. PiezoSleep requires that mice be single housed during recording. Therefore, separate cohorts were generated for the juvenile and adolescent groups in order to mitigate the lasting negative effects of social isolation during development ${ }^{20}$. We observed that Shank $3^{\Delta \mathrm{C} / \Delta \mathrm{C}}$ mice show a significant reduction in total daily sleep compared to WT littermates. Reduced sleep was significant in males and females, at both the juvenile and adolescent ages, and was seen in the light and dark phases (Fig.1A-D). Reductions in sleep bout length in the light and dark phase were also seen in adolescent male and female Shank $3^{\Delta \mathrm{C} / \Delta \mathrm{C}}$ (Fig. 1C and D). Our measurements did not reveal any significant differences in sleep amount or bout length between WT littermates and Shank3 ${ }^{\mathrm{WT} / \Delta \mathrm{C}}$ heterozygotes in either sex or age (Fig. 1A-D). Overall, the data show that male and female Shank $3^{\Delta \mathrm{C} / \Delta \mathrm{C}}$ mice have reduced total sleep as an early phenotype, and that sleep additionally becomes fragmented as the animals enter adolescence. The worsening of sleep symptoms during adolescence in Shank $3^{\Delta \mathrm{C} / \Delta \mathrm{C}}$ homozygous mice matches observations obtained from some Phelan-McDermid syndrome patients ${ }^{18,21}$.

\section{Causal effects of developmental sleep disruption in genetically vulnerable Shank $3^{\mathrm{WT} / \Delta \mathrm{C}}$ heterozygotes}

In contrast to homozygous Shank3 ASD mouse models, clinically relevant Shank3 heterozygotes have repeatedly been shown to have no or mild ASD relevant phenotypes ${ }^{13-17}$, in agreement with our sleep measurements (Fig. 1). We speculated that heterozygous mutation of Shank3 confers a clear genetic vulnerability in mice that may be sensitive to environmental perturbations during early postnatal life. A major question in the ASD field is whether sleep disruption commonly seen in patients, contributes to other ASD phenotypes. Additionally, ASD is diagnosed with an $\sim 4: 1$ male bias, although the basis for vulnerability in males or resilience in 
females is not understood 22,23 . Therefore, we reasoned that Shank3 heterozygous mice are an ideal model to examine gene $x$ sex $x$ environment interactions in ASD susceptibility.

Based on the recent publication of Jones and colleagues ${ }^{8}$, we tested the lasting effects of early life sleep disruption (ELSD) on WT and Shank3 ${ }^{\mathrm{WT} / \Delta \mathrm{C}}$ littermates. To specifically investigate whether early life represents a unique period of vulnerability to sleep disruption, we also tested the effects of post-adolescent sleep disruption (PASD) (Fig. 2A). Mice were treated by 7 days of sleep disruption using an automated method previously validated in multiple rodent species at both adult and developmental ages ${ }^{8,24,25}$. The home cage is placed on an orbital shaker set to rotate at 110 RPM for 10 seconds every 109 seconds (10 seconds on, 99 seconds off), producing a mild but frequent stimulus resulting in experimentally induced chronic sleep fragmentation $^{8}$. For ELSD treatments, litters of mouse pups were exposed to sleep disruption from P14-P21, together with their dam (WT) (Fig. 2A), as recently described using a prairie vole model $^{8}$. This period encompasses a clear consolidation of sleep stability, a shift in the relative proportions of rapid eye movement (REM) sleep and non-REM (NREM) sleep, and the emergence of a homeostatic sleep drive ${ }^{1,2}$, suggesting that this is a critical period in the development of sleep behavior. For PASD, separate cages of weaned males and females were exposed to sleep disruption from P56-P63 (Fig. 2A). Control groups were placed onto identical orbital shakers that were left off. Each group included male and female WT and Shank $3^{\mathrm{WT} / \Delta \mathrm{C}}$ heterozygous littermates. Because the dam is also experiencing sleep disruption in the ELSD experiment, pups were examined and weighed daily to ensure that they were receiving sufficient maternal care. Control and ELSD pups maintained healthy appearance and gained weight similarly with control pups (Fig. 2B), with the exception of the male Shank $3^{\mathrm{WT} / \Delta \mathrm{C}}$ group that gained slightly more weight than control; however, the weight in this group normalized after weaning (Fig 2 - figure supplement 1). Finally, to test whether ELSD treatment induces high levels of stress, a separate cohort of mice underwent control or ELSD treatment followed immediately by sacrifice at P21 and measurement of serum corticosterone by ELISA. Similar to what has been reported for prairie voles ${ }^{8}$, we did not observe any changes in corticosterone levels between ELSD pups and control (Fig. 2C, and Fig 2 - figure supplement 1), suggesting that pups acclimate to this treatment without sustained levels of stress.

Upon reaching P70, control, ELSD and PASD mice underwent an extensive panel of behavioral testing that lasted 6-7 weeks (Fig. 2A). We asked whether ELSD/PASD treatment caused lasting changes in behavior, whether Shank $3^{\mathrm{WT} / \Delta \mathrm{C}}$ heterozygotes were more vulnerable to these treatments than WT siblings, and whether distinct effects were seen between the sexes. In the control treatment group, we did not observe any differences in behavior between WT and Shank $3^{\mathrm{WT} / \Delta \mathrm{C}}$ littermates (Figs. 3 and 4), consistent with several previous reports ${ }^{13-17}$, suggesting that loss of a single copy of Shank3 alone is insufficient to induce robust ASD relevant phenotypes in mice. Strikingly, we observed that ELSD treatment, but not PASD treatment, resulted in lasting changes in behavior in genetically vulnerable Shank ${ }^{\mathrm{WT} / \Delta \mathrm{C}}$ heterozygotes, while WT littermates were resistant. Importantly, males and females showed completely distinct and non-overlapping behavioral changes (Fig. 3, male specific; Fig. 4, female specific). Following our behavior panel, these mice underwent a final sleep recording as in Fig. 1. We found that ELSD/PASD treatments had no lasting effect on sleep (Fig 2 - figure supplement 2), thus ruling out a sustained change in sleep as a driver of the altered behaviors.

Male Shank $3^{\mathrm{WT} / \Delta \mathrm{C}}$ heterozygotes exposed to the ELSD treatment exhibited reduced social approach behavior in the 3-chamber choice task, showing no preference for the social stimulus (S) in comparison to the non-social stimulus (N) (Fig. 3A-B). These mice also showed reduced paired-pulse inhibition of acoustic startle (PPI) (Fig. 3D), and hypo-activity in the open field test (Fig 3F). In these tasks, females showed comparable behaviors between Shank3 heterozygotes 
and WT siblings (Fig. 3C, E, and G). All groups performed comparably on the accelerating rotarod assay of motor coordination/learning, in the hidden-food assay of olfactory ability, in the marble burying assay for perseverative responses (Fig. 3 -figure supplement 1), and showed no changes in anxiety-like measures in the open field test (Fig. 3 -figure supplement 2). Therefore, hypo-activity in open-field, and reduced PPI and social approach behavior in ELSD-

Shank $3^{\mathrm{WT} / \Delta \mathrm{C}}$ males were unlikely to result directly from altered motor behavior, olfaction, or anxiety. Importantly, no changes in social approach, PPI, and open-field behavior were seen between WT and Shank3 ${ }^{\mathrm{WT} / \Delta \mathrm{C}}$ heterozygotes in the PASD group, suggesting that Shank3 ${ }^{\mathrm{WT} / \Delta \mathrm{C}}$ mice gain resilience to the effects of sleep disruption with maturation (Fig. 3). Together, these results clearly show that ELSD treatment interacts with Shank3 mutation to cause lasting changes in multiple behaviors in genetically vulnerable Shank $3^{\mathrm{WT} / \Delta \mathrm{C}}$ heterozygous males.

A female specific effect was observed in the elevated plus maze, typically considered an assay of anxiety-like behavior. Interestingly, Shank $3^{\mathrm{WT} / \Delta \mathrm{C}}$ females exposed to ELSD treatment showed a significant increase in time spent in the open arms of the plus maze in comparison to WT littermates, and a similar trend in the number of open arm entries (Fig. 4A and B). We describe this phenotype as "decreased risk aversion". No changes were seen in the control treatment group or any male groups (Fig. 4). PASD treatment resulted in a trend of increased time in the open arms of the elevated plus maze (Fig. 4A), suggesting that Shank $3^{W T / \Delta C}$ females may have an extended period of vulnerability to the effects of sleep loss. Overall, these findings show that early life sleep disruption can lead to behavioral changes in both male and female Shank ${ }^{\mathrm{WT} / \Delta \mathrm{C}}$ mice.

\section{Discussion}

Sleep disruption is a common comorbidity affecting individuals with ASD and other developmental disorders, including Phelan-McDermid syndrome ${ }^{5,6,12,18}$. A major question in the field is whether sleep disruption, particularly during development, is a driver of other phenotypes in ASD. We show that sleep disruption emerges as a developmental phenotype in Shank $3^{\Delta \mathrm{C} / \Delta \mathrm{C}}$ homozygotes of both sexes, but not in the clinically relevant Shank $3^{\mathrm{WT} / \Delta \mathrm{C}}$ heterozygotes (Fig. 1). Additionally, several studies have demonstrated behavioral phenotypes in Shank3 homozygous mouse models, that are largely absent from Shank3 heterozygotes ${ }^{13-18}$, similar to our observations of Shank3 heterozygotes reared under control conditions (Figs. 3 and 4). We speculate intrinsic developmental sleep disruption in the Shank3 homozygous mutants may be an important factor in the more severe behavioral phenotypes reported in this model. However, a causal relationship is difficult to establish without a means to robustly reverse the early sleep disruption. By using experimentally induced sleep disruption during targeted periods of postnatal life, we are now able to establish a causal role for early life sleep disruption in driving lasting and sex-specific changes in behavior in genetically vulnerable Shank3 heterozygous mice. Whether developmental sleep disruption similarly interacts with other ASD risk genes remains to be determined.

ASD is diagnosed with a clear male bias of $\sim 4: 1$, based on the core features of altered social interaction, communication deficits, and abnormal restricted/repetitive behavior. The basis of vulnerability in males, or resilience in females, that underlies this clear sex bias is not known ${ }^{23}$. However, it has been suggested that the genetic and environmental vulnerabilities underlying ASD susceptibility may in fact affect males and females in equal numbers, but that the phenotypic presentations diverge. Consequently, many affected females escape ASD diagnosis, as the diagnostic criteria are based on typical male presentation ${ }^{22,26,27}$. Unfortunately, this may lead to a lack of treatment in affected females. Our results show that Shank3 mutation 
and developmental sleep disruption interact to drive sex-specific changes in behavior, supporting the idea that the sexes may share vulnerability, but diverge in the phenotypic outcomes. Overall, this work points towards early life sleep as a risk factor in ASD susceptibility in both sexes.

\section{Materials and Methods}

\section{Mice}

All animal procedures were approved by the Institutional Animal Care and Use Committee of the University of North Carolina at Chapel Hill and were performed in accordance with the guidelines of the U.S. National Institutes of Health. Shank3 $\Delta \mathrm{C}$ mice (deletion of Shank3 exon 21 , described in Kouser et al., 2013 ${ }^{15}$ ) were generously provided by Dr. Paul Worley (Johns Hopkins University) and are maintained on a C57BL/6J background. Wild-type C57BL/6J breeders were regularly purchased from Jackson laboratories (strain \#000664). For sleep recording experiments described in Fig. 1, Shank $3^{\mathrm{WT} / \Delta \mathrm{C}}$ heterozygous males and females were crossed to generate cohorts containing WT, Shank $3^{\mathrm{WT} / \Delta \mathrm{C}}$ heterozygotes, and Shank $3^{\Delta \mathrm{C} / \Delta \mathrm{C}}$ homozygotes of both sexes. Separate cohorts were generated for sleep recordings for the juvenile (P25-P41) and adolescent (P42-P56) age groups. For the remainder of the experiments (Figs. 2-4), Shank ${ }^{\mathrm{WT} / \Delta \mathrm{C}}$ heterozygous sires were crossed with WT C57BL/6J dams to generate cohorts containing WT and Shank $3^{\mathrm{WT} / \Delta \mathrm{C}}$ heterozygotes of both sexes.

\section{Sleep behavior recordings}

Sleep recordings were conducted in a dedicated sleep behavior room free from any other mouse husbandry activities to allow for uninterrupted behavior assessment, and were maintained on a 12 hours:12 hours light:dark cycle (lights on 7am to 7pm). Sleep/wake behavior was recorded using a non-invasive home-cage monitoring system, PiezoSleep 2.0 (Signal Solutions, Lexington KY). The system uses a piezoelectric mat underneath the cage to detect vibrational movement of the mouse. Customized software (SleepStats, Signal Solutions, Lexington $\mathrm{KY}$ ) uses an algorithm to process the signal and discern sleeping respiratory patterns from waking respiratory patterns. Sleep was characterized primarily by periodic $(2-3 \mathrm{~Hz})$ and regular amplitude signals, which is typical of respiration from a sleeping mouse. In contrast, signals characteristic of wake were both the absence of characteristic sleep signals and higher amplitude, and irregular signals were associated with volitional movements, even subtle head movements during quiet wake. The piezoelectric signals in 2-s epochs were classified by a linear discriminant classifier algorithm based on multiple signal variables to assign a binary label of "sleep" or "wake." Data collected from the cage system were binned over specified time periods: $1 \mathrm{hr}$ bins to generate a daily sleep trace, $12 \mathrm{hr}$ bins for average light or dark phase percent sleep or sleep bout lengths, $24 \mathrm{hr}$ bin for total daily average percent sleep. To eliminate the impact of short and ambiguous arousals on the bout length statistics, a bout length count is initiated when a 30 -second interval contains greater than $50 \%$ sleep and terminates when a 30 second interval has less than $50 \%$ sleep. This algorithm has been validated by using electroencephalography, electromyography, and visual evaluation ${ }^{19,28,29}$. Male and female C57BL/6J wild type, Shank $3^{\mathrm{WT} / \Delta \mathrm{C}}$ heterozygous, Shank $3^{\Delta \mathrm{C} / \Delta \mathrm{C}}$ homozygous mice were individually housed in $15.5 \mathrm{~cm}^{2}$ cages with bedding, food and water. Mice were given two full dark cycles to acclimate to the recording cages before the beginning of data collection. No other animals were housed in the room during these experiments. 8-10 days of uninterrupted data was collected for baseline sleep behavior. Sleep amount and bout lengths were averaged from the multiple days of recordings for each individual prior to statistical comparisons between the experimental groups. 


\section{Early life and post-adolescent sleep disruption (ELSD/PASD)}

Shank $3^{\mathrm{WT} / \Delta \mathrm{C}}$ heterozygous mice and wild type littermates were subjected to 7days of 24-hour early life sleep disruption (ELSD, postnatal days $14-21$ ) or post adolescence sleep disruption (PASD, postnatal days $56-63$ ) using the methods described previously ${ }^{8}$. For sleep disruption the home cage was placed atop orbital shakers and mechanically agitated at $110 \mathrm{rpm}$ for 10 seconds following every 99 seconds still (109 seconds total per cycle), thus producing a mild but frequent mechanical stimulus, shown to interrupt sleep in rodents ${ }^{24,25}$. Control cages were kept atop unplugged orbital shakers and housed in the same room alongside the sleep disruption cohorts. For ELSD experiments each home cage contained 1 entire litter of mouse pups, including littermates of both sexes and genotypes, together with their dam. Eight litters were assigned to each of control and ELSD treatment across five cohorts, with an average of 7.6 pups per litter. Prior to start, all pups were confirmed capable of righting themselves immediately from a prone position and initial weights were taken. Individual weights were checked daily during treatment for all pups, and pups were tracked with non-toxic markings on their tails. Body condition of the pups and dams was also assessed daily and judged to be in good appearance. The ELSD treatment was well tolerated, no adverse effects were noted in any pups and no pups were excluded from further analysis. Dams were weighed on the first and last days of treatment. Following treatment, all litters were returned to the vivarium and pups were weaned, genotyped, and socially housed with same-sex littermates. For PASD experiments, young adults were weighed on the first and last days of treatment and visually assessed daily to confirm good body condition. Six litters were used, with an average of 7 mice per litter, and subjects were socially housed in groups of $3-5$ sex matched littermates. At the conclusion of the treatment, all animals were returned to the vivarium, prior to further behavioral characterization. Treatment took place in the room described above, with a 12:12 light cycle and no other animals in the room. Subjects were housed in clear standard mouse cages (Tecniplast) with irradiated cobb bedding, cotton nestlet, enrichment hut, and chow ad libitum. Hydrogel (ClearH2O) was supplied in place of water bottles to prevent splashing and was changed daily.

\section{Serum corticosterone quantification}

Control or ELSD treated mice aged P21 were briefly anesthetized with isoflurane, followed by euthanasia by cervical dislocation and decapitation. Trunk blood was collected and mixed with anti-coagulate ( $5 \%$ sodium citrate) to a final concentration of $1 \%$ sodium citrate. The blood and anti-coagulate mixture was centrifuged at 2000RCFs for 10 minutes. The supernatant (serum) was collected. Corticosterone level in the serum was assessed using the Arbor Assays corticosterone ELISA kit according to the manufacturer's instructions. Corticosterone concentration is calculated using a colorimetric readout in comparison with a standard curve measured with a BioTek Gen5 plate reader. Analysis was performed with the Arbor Assays Detect $X ®$ Corticosterone (OD) software. Results are expressed as $\mathrm{ng} / \mathrm{ml}$ of corticosterone concentration in mouse serum.

\section{Behavioral tests}

Mice were tested in a standardized battery of assays, conducted in the order listed below, using published methods ${ }^{30}$. More stressful procedures (acoustic startle test, buried food test following food deprivation) were carried out near the end of the regimen, to limit confounding effects of repeated testing. Mice were evaluated in only one or two different procedures per week.

Elevated plus maze. A 5-min test for anxiety-like behavior was carried out on the plus maze (elevation, $50 \mathrm{~cm} \mathrm{H}$; open arms, $30 \mathrm{~cm} \mathrm{~L}$; closed arms, $30 \mathrm{~cm} \mathrm{~L}$, walls, $20 \mathrm{~cm} \mathrm{H}$ ). Mice were placed in the center $(8 \mathrm{~cm} \times 8 \mathrm{~cm})$ at the beginning of the test. Measures were taken of percent open arm time and open arm entries. 
Open field. Exploratory activity was evaluated by a 1-hr test in a novel open field chamber (41 $\mathrm{cm} \mathrm{L}, 41 \mathrm{~cm} \mathrm{~W}, 30 \mathrm{~cm} \mathrm{H}$ ) crossed by a grid of photobeams (VersaMax system, AccuScan Instruments). Counts were taken of photobeam breaks in 5-min intervals, with separate measures for locomotor activity (total distance traveled) and vertical rearing movements. Anxiety-like behavior was assessed by measures of time spent in the center region.

Accelerating rotarod. Mice were first given 3 trials on the rotarod (Ugo Basile, Stoelting Co.), with $45 \mathrm{sec}$ between each trial. Two additional trials were conducted $48 \mathrm{hr}$ later, to evaluate consolidation of motor learning. In each trial, rpm (revolutions per minute) progressively increased from 3 to a maximum of $30 \mathrm{rpm}$. across $5 \mathrm{~min}$ (the maximum trial length), and latency to fall from the top of the rotating barrel was recorded.

Social approach in a 3-chamber choice test. Mice were evaluated for social motivation in a rectangular, 3-chambered box $(60 \mathrm{~cm} \mathrm{~L}, 41.5 \mathrm{~cm} \mathrm{~W}, 20 \mathrm{~cm} \mathrm{H})$ fabricated from clear Plexiglas. Dividing walls had doorways allowing access into each chamber. At the start of the test, the mouse was placed in the middle chamber and allowed to explore for $10 \mathrm{~min}$, with the doorways into the 2 side chambers open. After the habituation period, the test mouse was enclosed in the center compartment of the social test box, and an unfamiliar stranger (a sex-matched, adult C57BL/6J mouse) was placed in one of the side chambers. The stranger mouse was enclosed in a small Plexiglas cage drilled with holes, which allowed nose contact. An identical empty Plexiglas cage was placed in the opposite side of the chamber. Following placement of the stranger and the empty cage, the doors were re-opened, and the subject was allowed to explore the social test box for a 10-min session. An automated image tracking system (Noldus Ethovision) provided measures of time in spent within $5 \mathrm{~cm}$ proximity to each cage, and entries into each side of the social test box.

Marble-burying. Mice were tested for exploratory digging in a Plexiglas cage, placed inside a sound-attenuating chamber with ceiling light and fan. The cage floor had $5 \mathrm{~cm}$ of corncob bedding, with 20 black glass marbles ( $14 \mathrm{~mm}$ diameter) set up in a $5 \times 4$ grid on top of the bedding. Measures were taken of the number of marbles buried (i.e., at least two-thirds of the marble covered by bedding) at the end of the 30-min test.

Acoustic startle. Mice were evaluated for prepulse inhibition of acoustic startle responses in an automated piezoelectric-based system (SR-Lab, San Diego Instruments). The test had 42 trials (7 of each type): no-stimulus trials, trials with the acoustic startle stimulus (40 msec; $120 \mathrm{~dB}$ ) alone, and trials in which a prepulse stimulus (20 msec; either $74,78,82,86$, or $90 \mathrm{~dB}$ ) occurred $100 \mathrm{msec}$ before the onset of the startle stimulus. Levels of prepulse inhibition at each prepulse sound level were calculated as 100 - [(response amplitude for prepulse stimulus and startle stimulus together/response amplitude for startle stimulus alone) $x 100]$.

Buried food test. Mice were presented with an unfamiliar food (Froot Loops, Kellogg Co.) in the home cage several days before the test. All home cage food was removed 16-24 hr before the test. The assay was conducted in a tub cage $(46 \mathrm{~cm} \mathrm{~L}, 23.5 \mathrm{~cm} \mathrm{~W}, 20 \mathrm{~cm} \mathrm{H})$, containing paper chip bedding ( $3 \mathrm{~cm}$ deep). One Froot Loop was buried in the cage bedding, and mice were given 15 min to locate the buried food. Latency to find the food was recorded.

\section{Statistical analysis}

Data was analyzed in GraphPad Prism version 9.1.0 (GraphPad Software, LLC). All results are reported as mean +/- SEM. Sleep characterization was performed with 2-way ANOVA with genotype and time of day as factors, followed by Tukey's multiple comparisons test for 24-hour sleep amount, and 1-way ANOVA with Tukey's multiple comparisons test for light/dark cycle sleep amount and bout duration. Weights were compared using 2-way ANOVA and serum 
corticosterone was compared using two-tailed unpaired t-test. Behavioral assays were analyzed using multiple unpaired t-tests for genotype with Holm-Šídák method (Open Field Test, Elevated Plus Maze, Olfactory, Marble Burying), multiple paired t-tests for genotype with Holm-Šídák method (Sociability), and 2-way ANOVA with Šídák's multiple comparisons test with genotype and trial as factors (Rotarod, Pre-Pulse Inhibition). Experimental units and $\mathrm{n}$ are reported in figures and figure legends.

\section{Acknowledgements}

This work was supported by a Bridge to Independence Award to GHD from the Simons Foundation Autism Research Initiative (SFARI), and by the Mouse Behavioral Phenotyping Core of the UNC Intellectual and Developmental Disabilities Research Center (NICHD; P50 HD103573; PI: Joseph Piven). We wish to thank Dr. Paul Worley (Johns Hopkins University) for generously providing the Shank3 $\Delta$ C ASD mouse model. We wish to thank Dr. Carolyn Jones and Dr. Miranda Lim (Oregon Health Sciences University) for consultation and technical advice on establishing the early life sleep disruption paradigm based on their recent publication ${ }^{8}$.

\section{Competing Interests}

The authors declare no competing interests

\section{References}

1 Rensing, N., Moy, B., Friedman, J. L., Galindo, R. \& Wong, M. Longitudinal analysis of developmental changes in electroencephalography patterns and sleep-wake states of the neonatal mouse. PLoS One 13, e0207031, doi:10.1371/journal.pone.0207031 (2018).

2 Nelson, A. B., Faraguna, U., Zoltan, J. T., Tononi, G. \& Cirelli, C. Sleep patterns and homeostatic mechanisms in adolescent mice. Brain Sci 3, 318-343, doi:10.3390/brainsci3010318 (2013).

3 Frank, M. G. Sleep and synaptic plasticity in the developing and adult brain. Curr Top Behav Neurosci 25, 123-149, doi:10.1007/7854_2014_305 (2015).

4 Barone, I., Hawks-Mayer, H. \& Lipton, J. O. Mechanisms of sleep and circadian ontogeny through the lens of neurodevelopmental disorders. Neurobiol Learn Mem 160, 160-172, doi:10.1016/j.nlm.2019.01.011 (2019).

5 Mazzone, L., Postorino, V., Siracusano, M., Riccioni, A. \& Curatolo, P. The Relationship between Sleep Problems, Neurobiological Alterations, Core Symptoms of Autism Spectrum Disorder, and Psychiatric Comorbidities. J Clin Med 7, doi:10.3390/jcm7050102 (2018).

6 Missig, G., McDougle, C. J. \& Carlezon, W. A., Jr. Sleep as a translationally-relevant endpoint in studies of autism spectrum disorder (ASD). Neuropsychopharmacology, doi:10.1038/s41386-019-0409-5 (2019).

7 Veatch, O. J. et al. Shorter sleep duration is associated with social impairment and comorbidities in ASD. Autism Res 10, 1221-1238, doi:10.1002/aur.1765 (2017).

8 Jones, C. E. et al. Early-life sleep disruption increases parvalbumin in primary somatosensory cortex and impairs social bonding in prairie voles. Sci Adv 5, eaav5188, doi:10.1126/sciadv.aav5188 (2019). 
9 Kayser, M. S., Yue, Z. \& Sehgal, A. A critical period of sleep for development of courtship circuitry and behavior in Drosophila. Science 344, 269-274, doi:10.1126/science.1250553 (2014).

10 Durand, C. M. et al. Mutations in the gene encoding the synaptic scaffolding protein SHANK3 are associated with autism spectrum disorders. Nat Genet 39, 25-27, doi:10.1038/ng1933 (2007).

11 Costales, J. L. \& Kolevzon, A. Phelan-McDermid Syndrome and SHANK3: Implications for Treatment. Neurotherapeutics 12, 620-630, doi:10.1007/s13311-015-0352-z (2015).

12 Bro, D. et al. Sleep Disturbances in Individuals With Phelan-McDermid Syndrome: Correlation With Caregivers' Sleep Quality and Daytime Functioning. Sleep 40, doi:10.1093/sleep/zsw062 (2017).

13 Speed, H. E. et al. Autism-Associated Insertion Mutation (InsG) of Shank3 Exon 21 Causes Impaired Synaptic Transmission and Behavioral Deficits. J Neurosci 35, 96489665, doi:10.1523/JNEUROSCI.3125-14.2015 (2015).

14 Jaramillo, T. C. et al. Altered Striatal Synaptic Function and Abnormal Behaviour in Shank3 Exon4-9 Deletion Mouse Model of Autism. Autism Res 9, 350-375, doi:10.1002/aur.1529 (2016).

15 Kouser, M. et al. Loss of predominant Shank3 isoforms results in hippocampusdependent impairments in behavior and synaptic transmission. $J$ Neurosci 33, 1844818468, doi:10.1523/JNEUROSCI.3017-13.2013 (2013).

16 Drapeau, E., Riad, M., Kajiwara, Y. \& Buxbaum, J. D. Behavioral Phenotyping of an Improved Mouse Model of Phelan-McDermid Syndrome with a Complete Deletion of the Shank3 Gene. eNeuro 5, doi:10.1523/ENEURO.0046-18.2018 (2018).

17 Matas, E. et al. Major motor and gait deficits with sexual dimorphism in a Shank3 mutant mouse model. Mol Autism 12, 2, doi:10.1186/s13229-020-00412-8 (2021).

18 Ingiosi, A. M. et al. Shank3 modulates sleep and expression of circadian transcription factors. Elife 8, doi:10.7554/eLife.42819 (2019).

19 Mang, G. M. et al. Evaluation of a piezoelectric system as an alternative to electroencephalogram/ electromyogram recordings in mouse sleep studies. Sleep 37, 1383-1392, doi:10.5665/sleep.3936 (2014).

20 Kercmar, J., Budefeld, T., Grgurevic, N., Tobet, S. A. \& Majdic, G. Adolescent social isolation changes social recognition in adult mice. Behav Brain Res 216, 647-651, doi:10.1016/j.bbr.2010.09.007 (2011).

21 Kolevzon, A., Delaby, E., Berry-Kravis, E., Buxbaum, J. D. \& Betancur, C. Neuropsychiatric decompensation in adolescents and adults with Phelan-McDermid syndrome: a systematic review of the literature. Mol Autism 10, 50, doi:10.1186/s13229019-0291-3 (2019).

22 Lai, M. C., Lombardo, M. V., Auyeung, B., Chakrabarti, B. \& Baron-Cohen, S. Sex/gender differences and autism: setting the scene for future research. J Am Acad Child Adolesc Psychiatry 54, 11-24, doi:10.1016/j.jaac.2014.10.003 (2015).

23 Baron-Cohen, S. et al. Why are autism spectrum conditions more prevalent in males? PLoS Biol 9, e1001081, doi:10.1371/journal.pbio.1001081 (2011).

24 Sinton, C. M., Kovakkattu, D. \& Friese, R. S. Validation of a novel method to interrupt sleep in the mouse. $J$ Neurosci Methods 184, 71-78, doi:10.1016/j.jneumeth.2009.07.026 (2009).

$25 \mathrm{Li}, \mathrm{Y}$. et al. Effects of chronic sleep fragmentation on wake-active neurons and the hypercapnic arousal response. Sleep 37, 51-64, doi:10.5665/sleep.3306 (2014).

26 Wood-Downie, H. et al. Sex/Gender Differences in Camouflaging in Children and Adolescents with Autism. J Autism Dev Disord 51, 1353-1364, doi:10.1007/s10803-02004615-z (2021). 
27 Kreiser, N. L. \& White, S. W. ASD in females: are we overstating the gender difference in diagnosis? Clin Child Fam Psychol Rev 17, 67-84, doi:10.1007/s10567-013-0148-9 (2014).

28 Donohue, K. D., Medonza, D. C., Crane, E. R. \& O'Hara, B. F. Assessment of a noninvasive high-throughput classifier for behaviours associated with sleep and wake in mice. Biomed Eng Online 7, 14, doi:10.1186/1475-925X-7-14 (2008).

29 Flores, A. E. et al. Pattern recognition of sleep in rodents using piezoelectric signals generated by gross body movements. IEEE Trans Biomed Eng 54, 225-233, doi:10.1109/TBME.2006.886938 (2007).

30 Jimenez, J. A. et al. Chd8 haploinsufficiency impairs early brain development and protein homeostasis later in life. Mol Autism 11, 74, doi:10.1186/s13229-020-00369-8 (2020). 


\section{Figure legends}

Figure 1. Developmental sleep disruption in Shank3 $\Delta$ C ASD mouse models. (A-D) non-invasive home cage sleep recordings were conducted for male and female Shank $3^{\mathrm{WT} / \Delta \mathrm{C}}$ heterozygotes, Shank $3^{\Delta \mathrm{C} / \Delta \mathrm{C}}$ homozygotes, and wild-type (WT) littermates, at two developmental time points: juvenile (P23-P41), and adolescent (P42-P56). (A) juvenile males, (B) juvenile females, (C) adolescent males, (D) adolescent females. (A-D) Left panel: trace of daily average hourly percent sleep, dark phase indicated by gray box. Statistical differences for specific hours of the day and animal numbers for each genotype are indicated (2-way ANOVA with Tukey correction with time and genotype as factors). Center panel: average hourly percent sleep for the light or dark phase (12hrs), or the daily average sleep per hour. Right panel: average sleep bout length observed in the light or dark phase. Statistical differences are indicated. ${ }^{*} P<0.05,{ }^{* *} P<0.01$, ${ }^{* * *} \mathrm{P}<0.001$ (1-way ANOVA with Tukey's multiple comparisons test). Error bars indicate \pm SEM.

Figure 2. Early life and post adolescent sleep disruption. (A) Experimental design. Cohorts of mice undergo 7 days of sleep disruption from P14-P21: early life sleep disruption (ELSD), or from P56-P63: post adolescent sleep disruption (PASD). Control cohorts are left undisturbed. Control, ELSD, and PASD cohorts include male and female WT and Shank $3^{\mathrm{WT} / \Delta \mathrm{C}}$ heterozygous littermates. Upon reaching adulthood (P70) control, ELSD, and PASD cohorts undergo a panel of behavioral testing lasting 6-7 weeks. (B) Control and ELSD pups were weighed daily from P14-P21. No differences were seen between groups. N=37 control pups; 39 ELSD pups. (C) Separate cohorts of control and ELSD treatment were sacrificed at P21 and serum corticosterone (cort.) was measured using ELISA. No differences were seen between groups. $\mathrm{N}=17$ control, 33 ELSD. Error bars indicate \pm SEM.

Figure 3. ELSD drives male-specific changes in behavior in Shank3 ${ }^{\mathrm{WT} / \Delta \mathrm{C}}$ heterozygotes. (A) Examples of individual WT and Shank $3^{\mathrm{WT} / \Delta \mathrm{C}}$ heterozygotes (HET) from control, ELSD, and PASD treatment groups performing the 3-chamber social approach task. Heat map indicates time in location. Mice can freely move between 3-chambers and choose to spend time engaging with the non-social stimulus (N) or with a social stimulus (S). (B-C) Time spent in proximity with the non-social (N) and social stimulus (S) for WT and HET males (B) or females (C). ELSD HET males show no preference for the social stimulus. (D-E) Percent prepulse inhibition (PPI) of acoustic startle responses for WT and HET males (D) or females (E). ELSD HET males show reductions in PPI response. (F-G) Total distance traveled in the open-field task for WT and HET males $(F)$ or females $(G)$. ELSD HET males show hypo-activity in the open field test. ${ }^{*} P<0.05$, ${ }^{* *} \mathrm{P}<0.01,{ }^{* *} \mathrm{P}<0.001$ (Sociability, paired t-test with Holm-Šídák correction; PPI, 2-way ANOVA with Šídák's multiple comparisons test: Open field, unpaired t-test with Tukey's multiple comparisons). Error bars indicate \pm SEM. N=8-12 mice per group/sex/genotype.

Figure 4. ELSD drives female-specific changes in elevated plus maze behavior in Shank $3^{\mathrm{WT} / \Delta \mathrm{C}}$ heterozygotes. (A-D) Percent open arm time and entries for WT and Shank $3^{\mathrm{WT} / \Delta \mathrm{C}}$ heterozygotes (HET) from control, ELSD, and PASD treatment groups. (A) Female \% time spent in the open arms. ELSD HET females spend significantly more time in the open arms than WT littermates. (B) Female \% open arms entries. (C) Male \% time spent in the open arms. (D) Male \% open arm entries. ${ }^{* *} \mathrm{P}<0.01$ (unpaired t-test for genotype with Tukey's multiple comparisons test). Error bars indicate \pm SEM. $\mathrm{N}=8-12$ mice per group/sex/genotype.

\section{Supplementary figures}

Figure 2-figure supplement 1. Weight gain and corticosterone levels in control and ELSD cohorts. (A-B) Control and ELSD cohorts were weighed daily from P14-P21 to ensure that pups 
were receiving sufficient maternal care during ELSD treatment, and at multiple time points during the remainder of the experiment. (A) weight of male control and ELSD cohorts. Shank $3^{\mathrm{WT} / \Delta \mathrm{C}}$ heterozygotes (HET) exposed to ELSD gain weight faster than WT littermates; however, this difference was normalized by 5 weeks of age. $\mathrm{N}=8-11$ per treatment/genotype. ${ }^{*} \mathrm{P}<0.05,{ }^{*} \mathrm{P}<0.01$ (2-way ANOVA). (B) Weight of female control and ELSD cohorts. No differences in weight between treatments or genotypes was observed. $\mathrm{N}=8-12$ per treatment/genotype. (C) Serum corticosterone (cort.) was measured using ELISA from separate cohorts of control and ELSD treated pups at P21. No differences in cort. levels between treatments or genotypes were observed.

Figure 2-figure supplement 2. ELSD/PASD treatments do not result in any sustained changes in sleep behavior. (A-F) Following completion of the behavioral characterization, non-invasive home cage sleep recordings were conducted for male and female Shank $3^{\mathrm{WT} / \Delta \mathrm{C}}$ heterozygotes (HET), and wild-type (WT) littermates, exposed to control, ELSD, and PASD treatments. (A) control males, (B) ELSD males, (C) PASD males, (D) control females, (E) ELSD females, (F) PASD females. (A-F) Left panel: trace of daily average hourly percent sleep, dark phase indicated by gray box. Center panel: average hourly percent sleep for the light or dark phase (12hrs), or the daily average sleep per hour. Right panel: average sleep bout length observed in the light or dark phase. ${ }^{*} \mathrm{P}<0.05$, (2-way ANOVA with Tukey correction). Error bars indicate \pm SEM. $\mathrm{N}=8-12$ mice per group/sex/genotype.

Figure 3-figure supplement 1. ELSD/PASD treatment did not result in any lasting changes in the accelerating rotarod, marble burying, or hidden food assays. (A-B) Latency to fall in the accelerating rotarod assay of motor coordination and motor learning in control, ELSD, and PASD treated WT and Shank $3^{\mathrm{WT} / \Delta \mathrm{C}}$ heterozygous (HET) males $(\mathrm{A})$ and females $(\mathrm{B})$. No differences were observed. (C-D) Marbles buried by control, ELSD, and PASD treated males (C) and females (D). No differences were observed. (E-F) Latency to find hidden food in the olfaction test. No differences were observed. $\mathrm{N}=8-12$ per treatment/sex/genotype.

Figure 3-figure supplement 2. No changes in anxiety-like measures in the open field test. (A-B) no differences observed in total time spent in the center of the open field from control, ELSD, and PASD treated WT and Shank $3^{\mathrm{WT} / \Delta \mathrm{C}}$ heterozygous (HET) males (A) and females (B). (C-D) no differences observed in number of rears during the open field test from control, ELSD, and PASD treated males $(C)$ and females (D). N=8-12 per treatment/sex/genotype. 


\section{Figure 1}
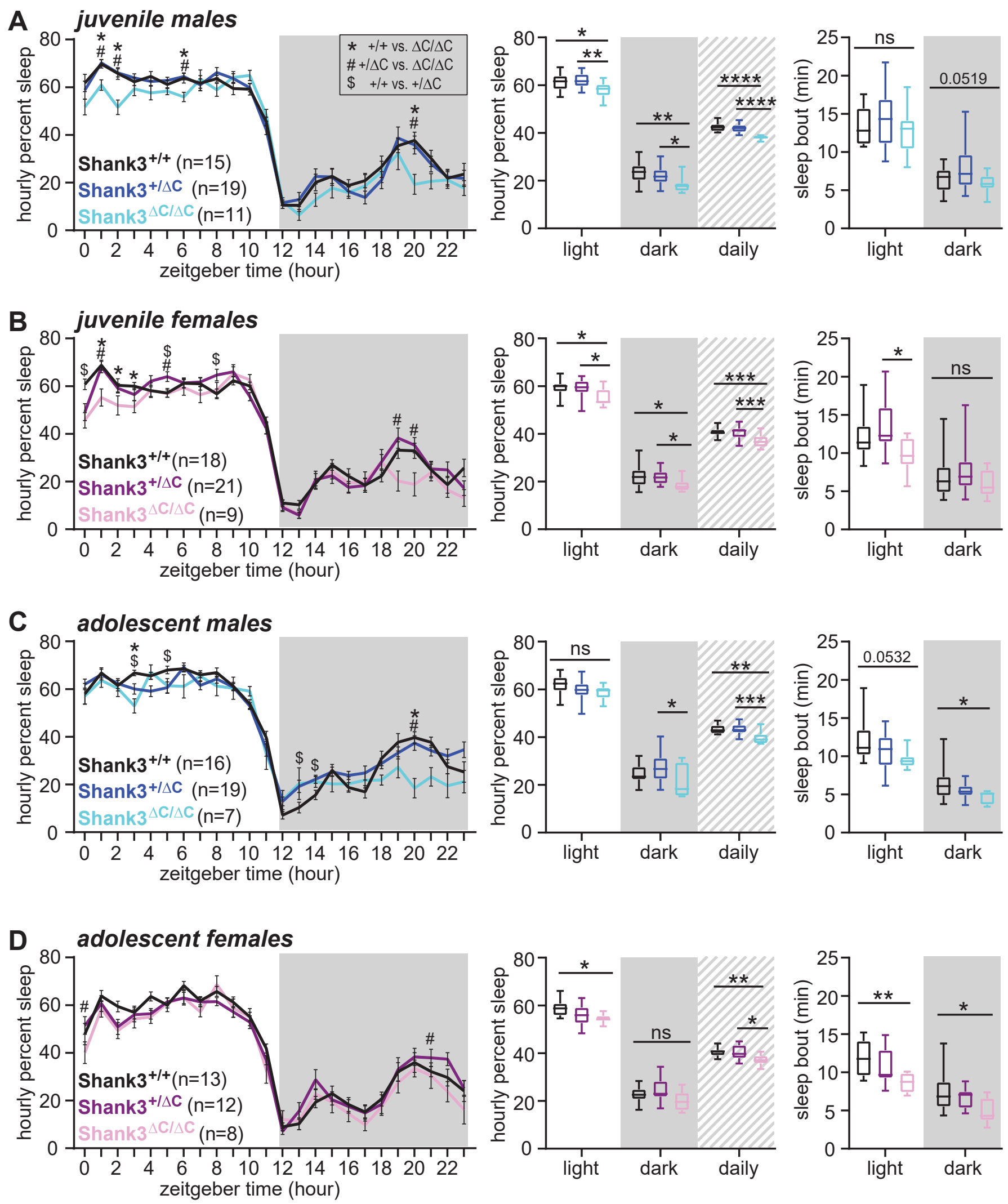


\section{Figure 2}
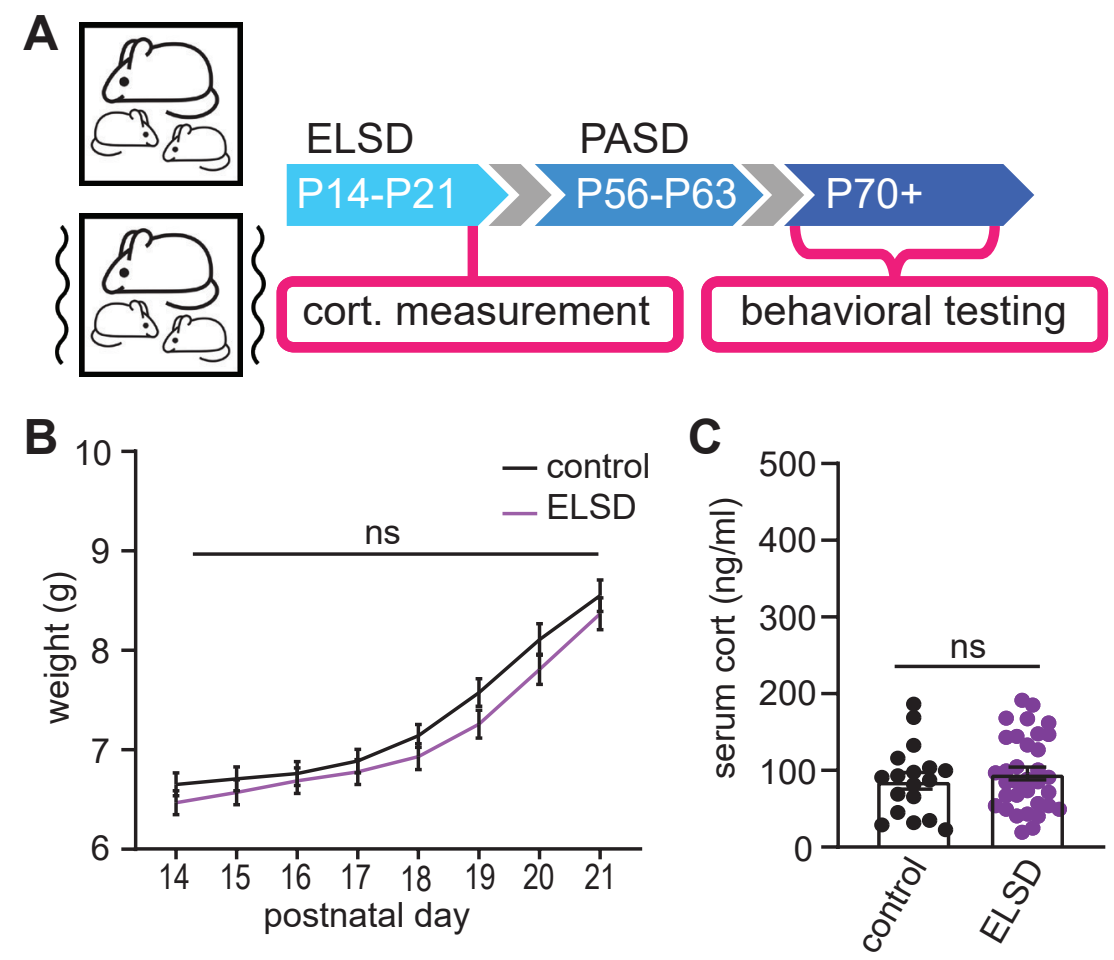
bioRxiv preprint doi: https://doi.org/10.1101/2021.09.23.461518; this version posted September 24, 2021. The copyright holder for this preprint Figure (3hich was not certified by peer review) is the author/funder. All rights reserved. No reuse allowed without permission.

A

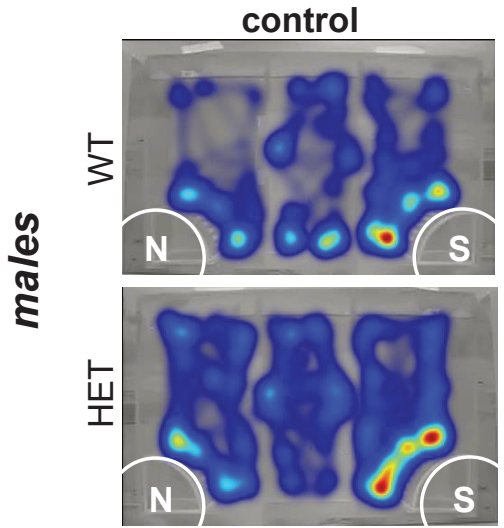

B

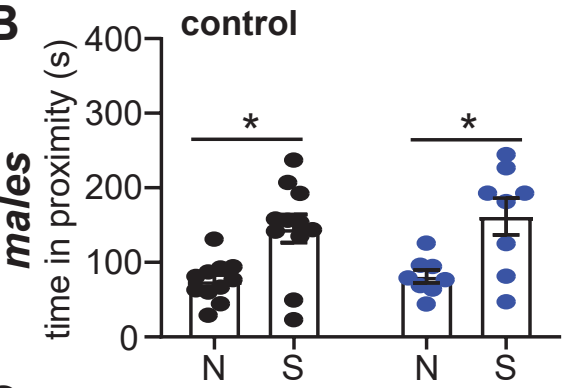

C

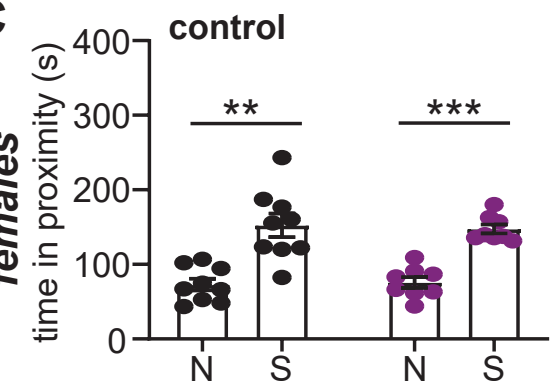

D

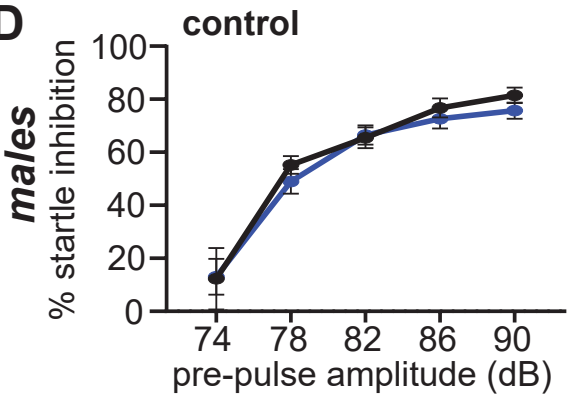

E

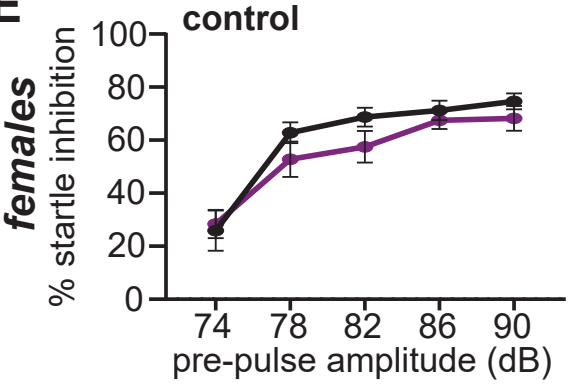

ELSD
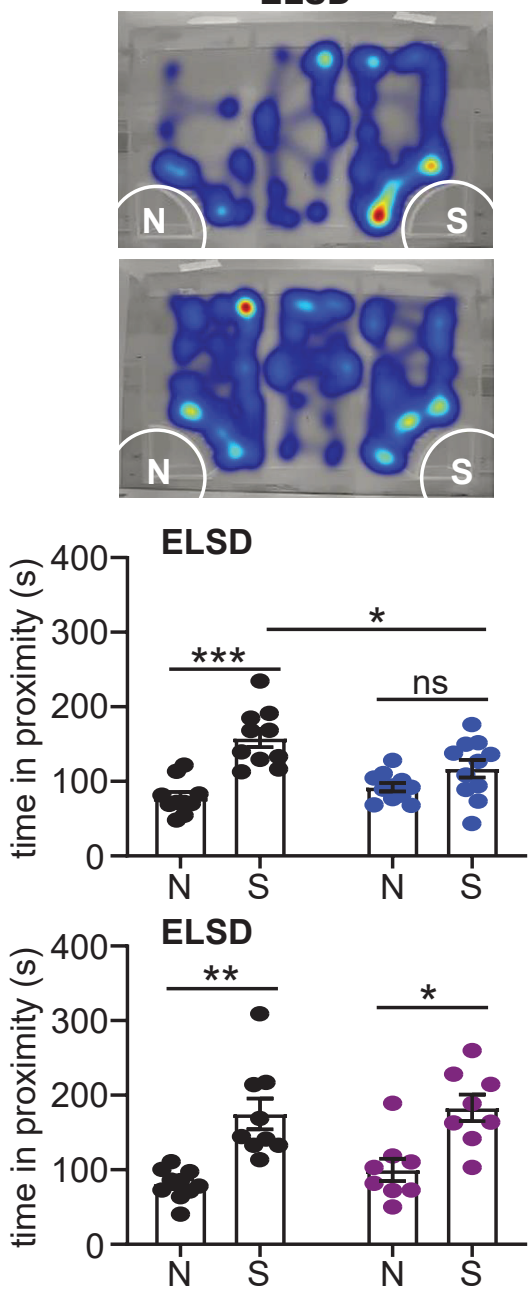

ELSD
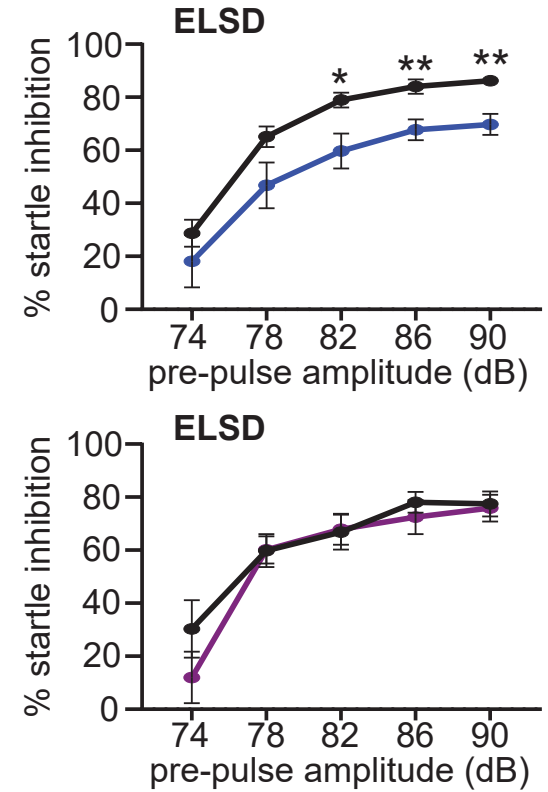

PASD
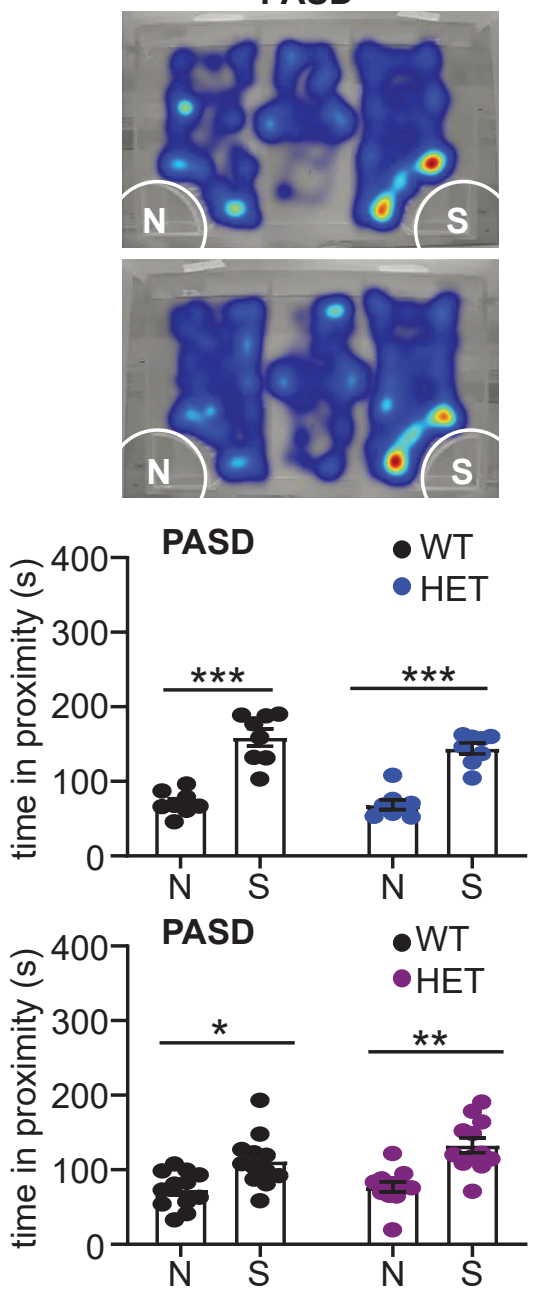

PASD

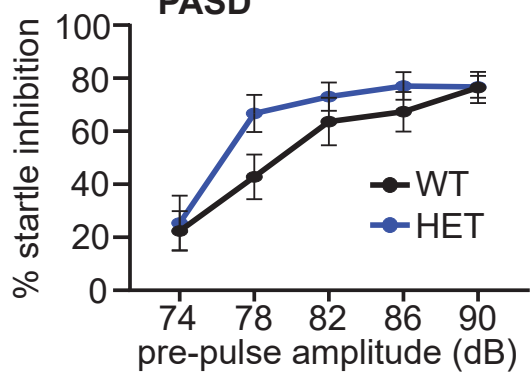

PASD

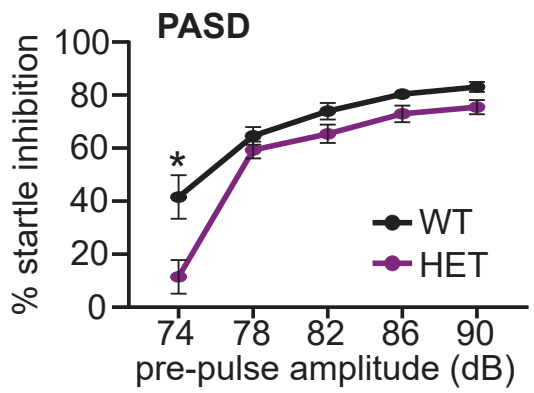

F

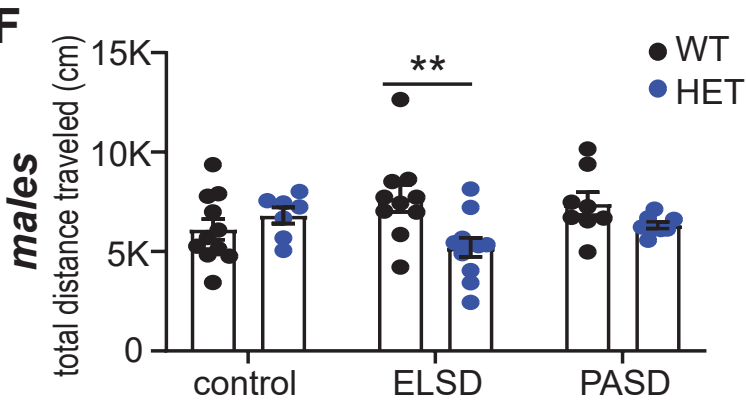

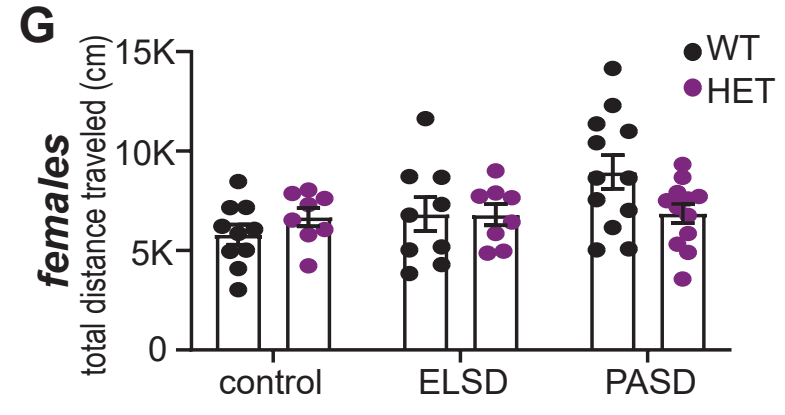


Figure 4

A

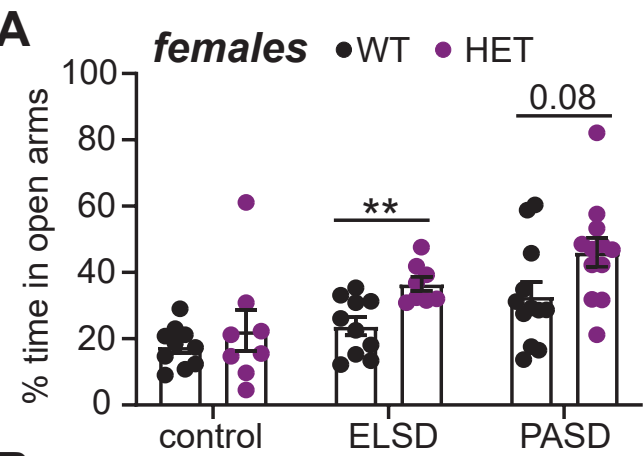

B

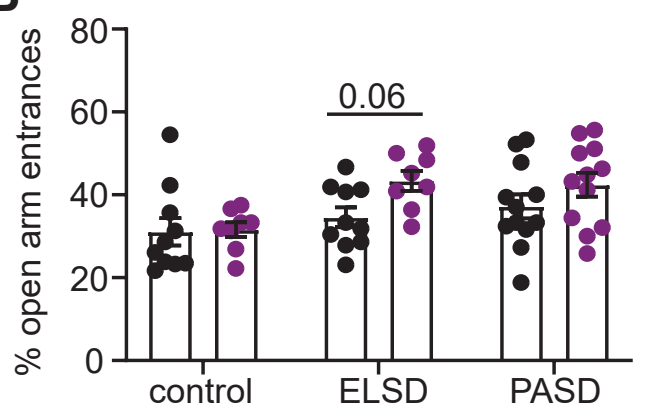

C

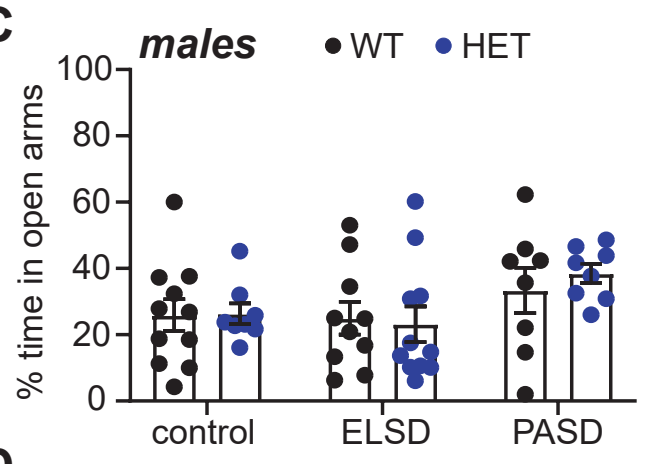

D

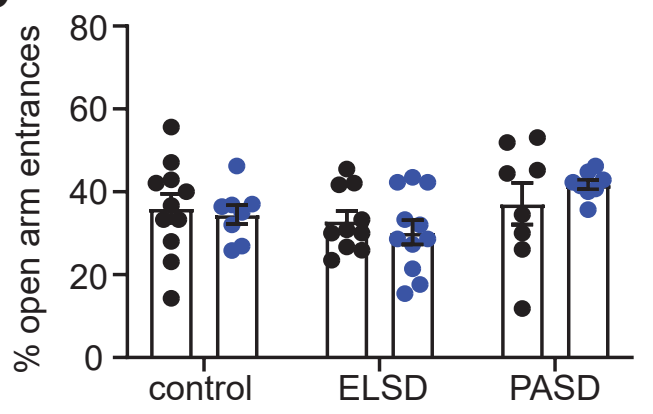




\section{A males}
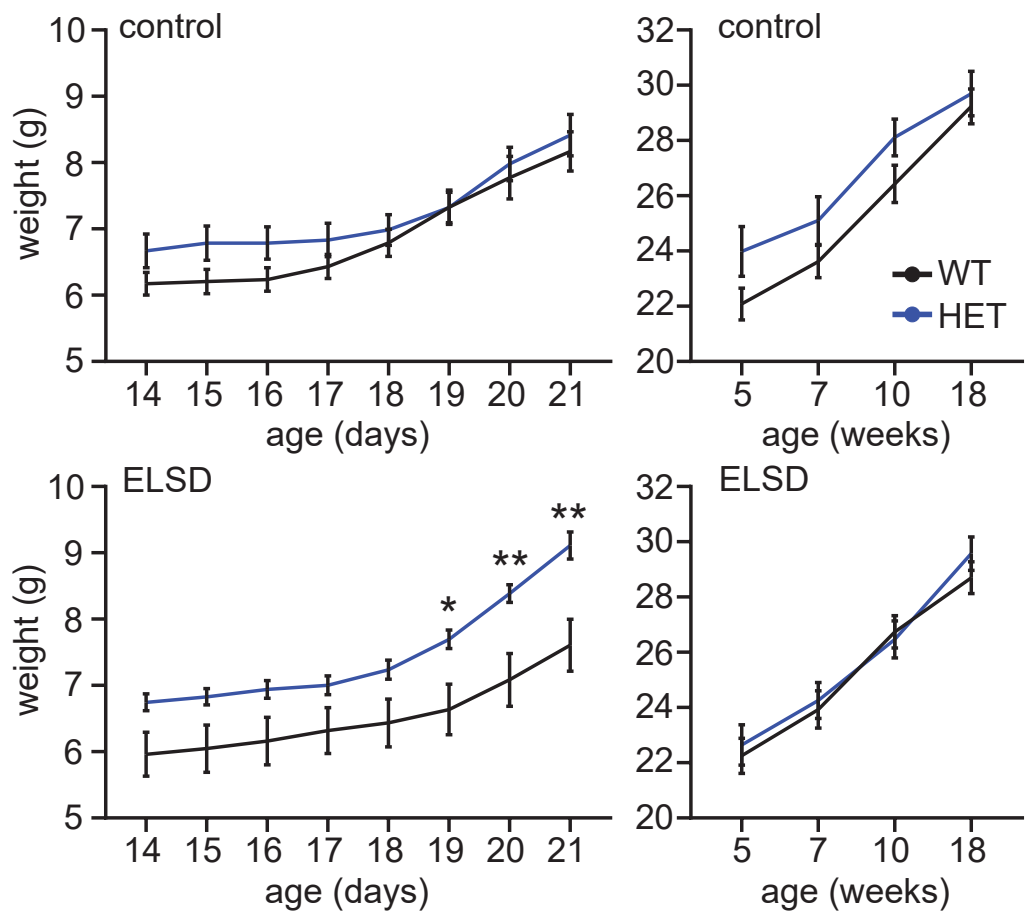

B

females
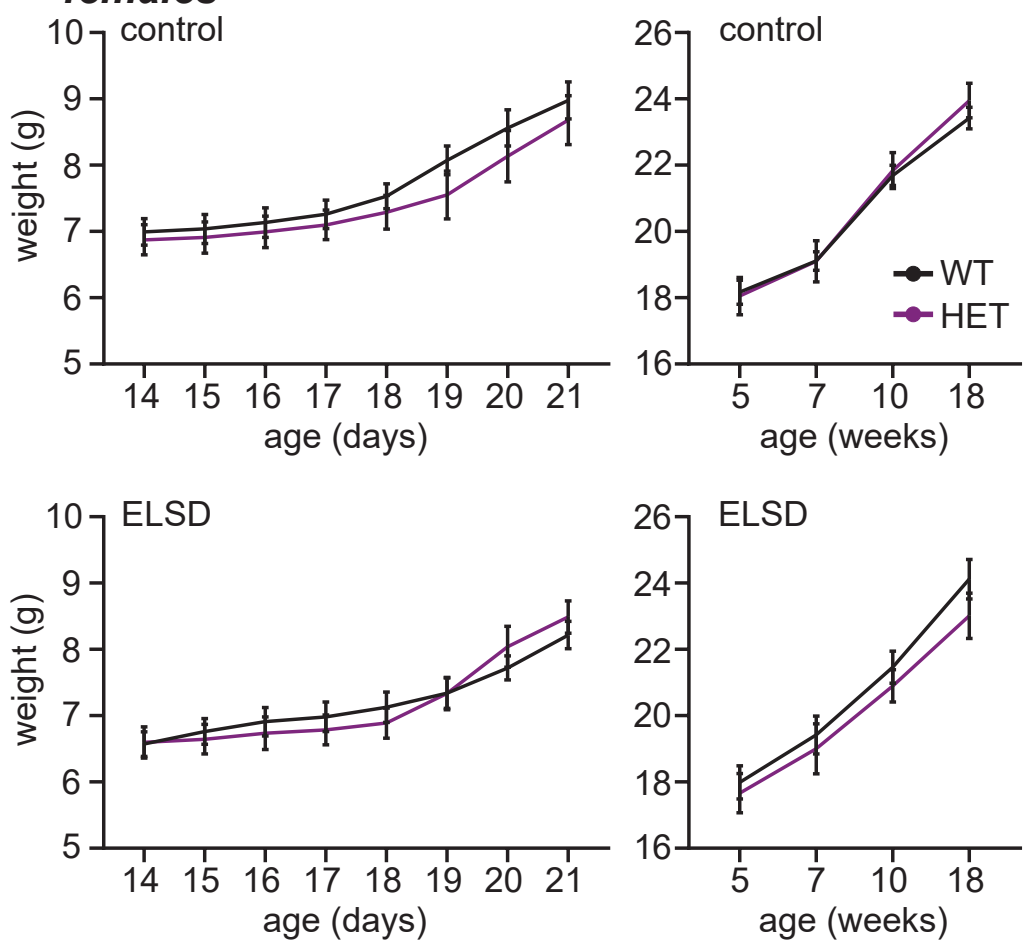

C
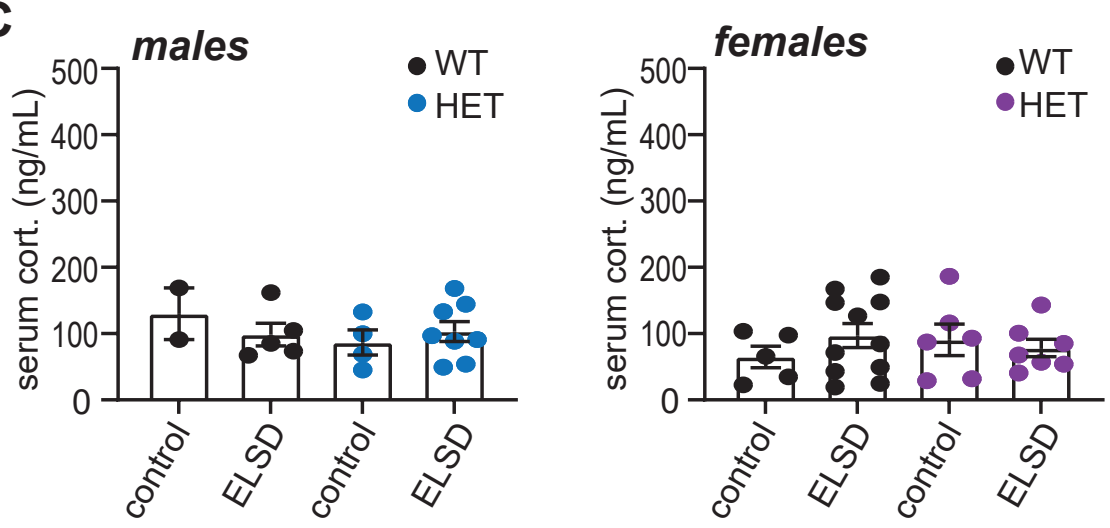
bioRxiv preprint doi: https://doi.org/10.1101/2021.09.23.461518; this version posted September 24, 2021. The copyright holder for this preprint

Figurevack figgureesitupp plemeqniev is the author/funder. All rights reserved. No reuse allowed without permission.

A

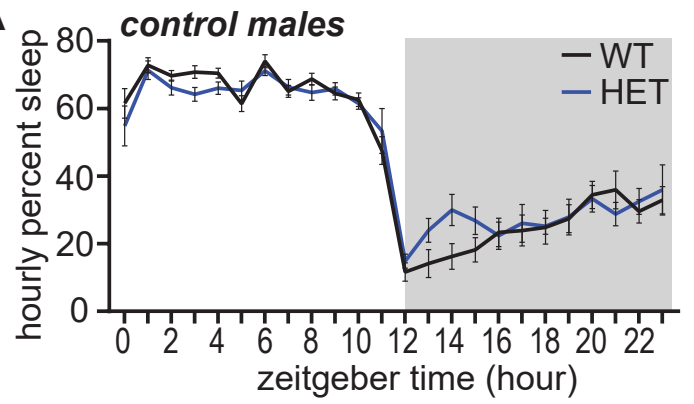

B

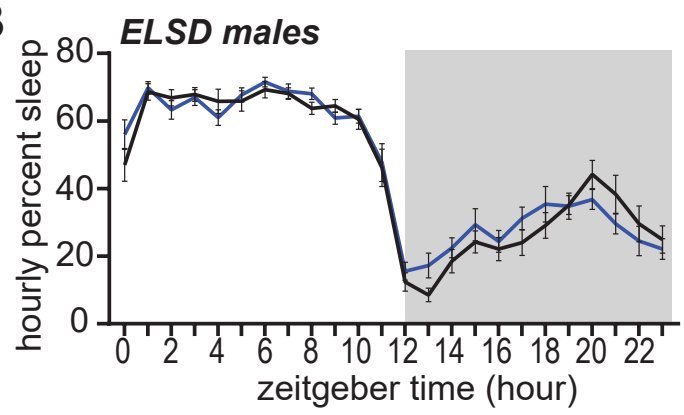

C

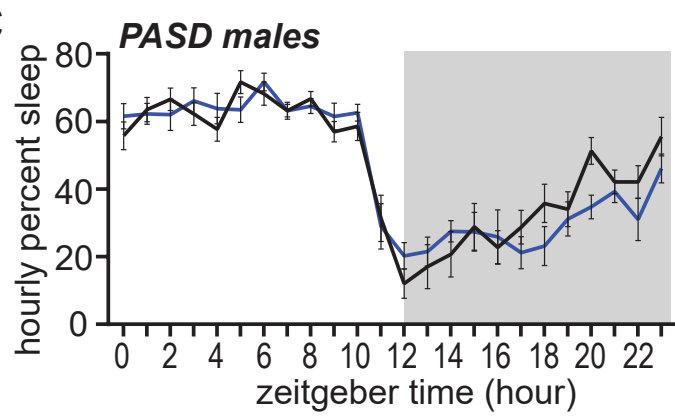

D

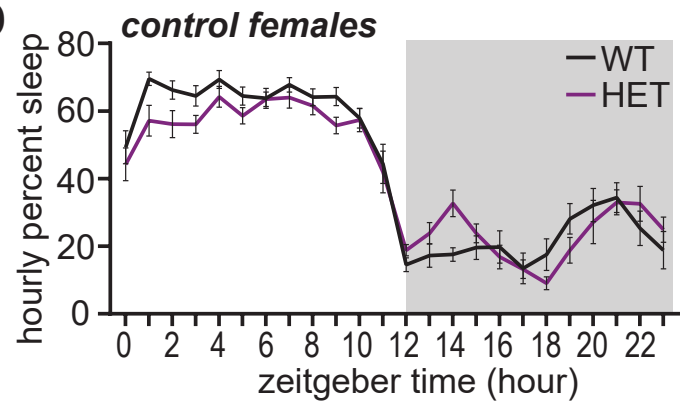

E

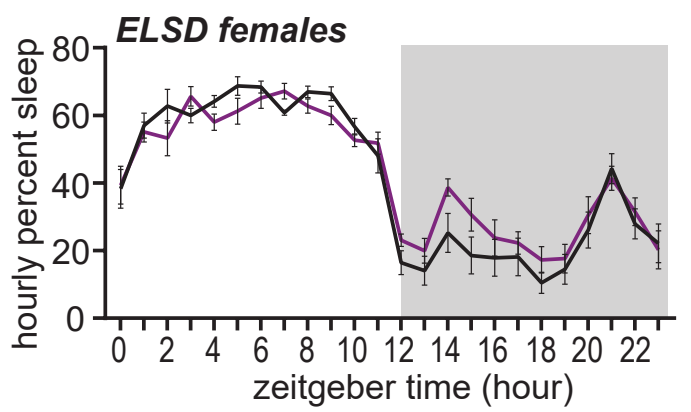

F

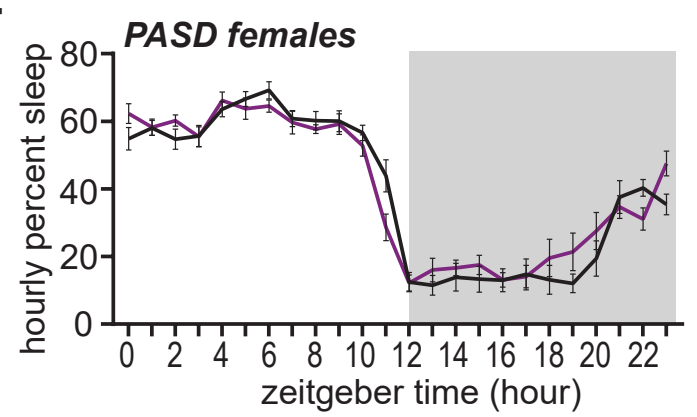

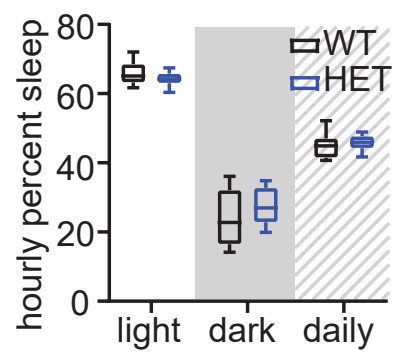
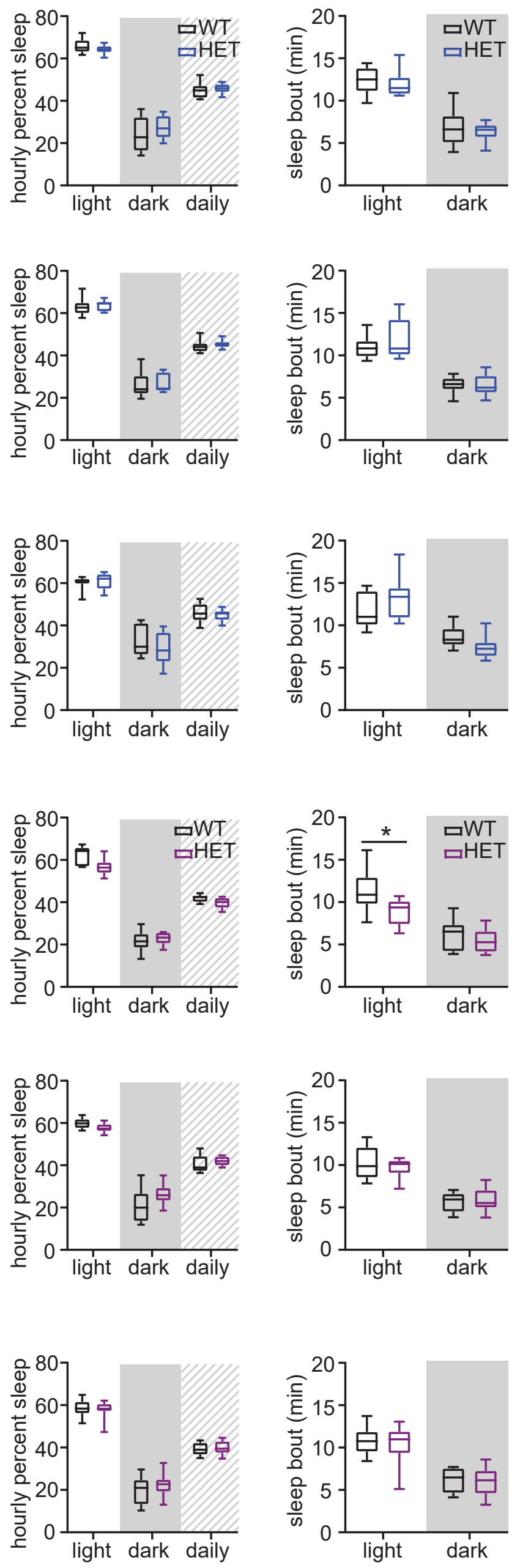
A accelerating rotarod
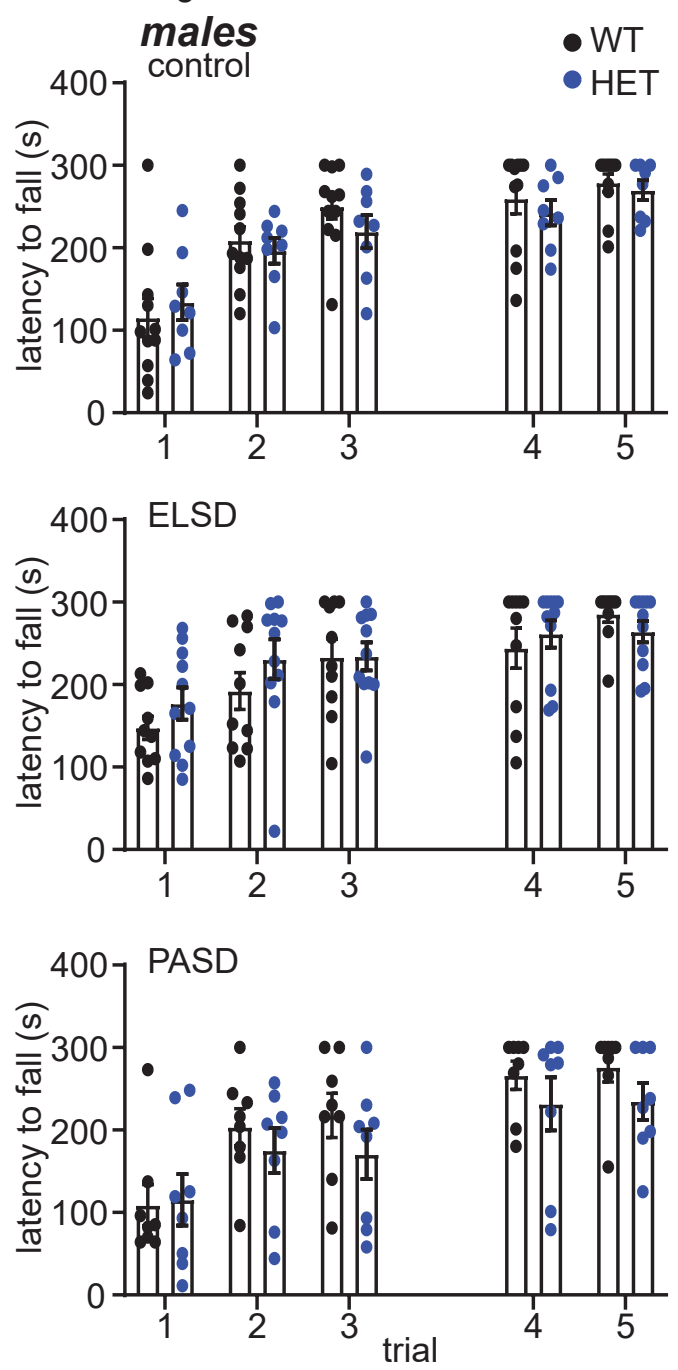

C marble burying assay

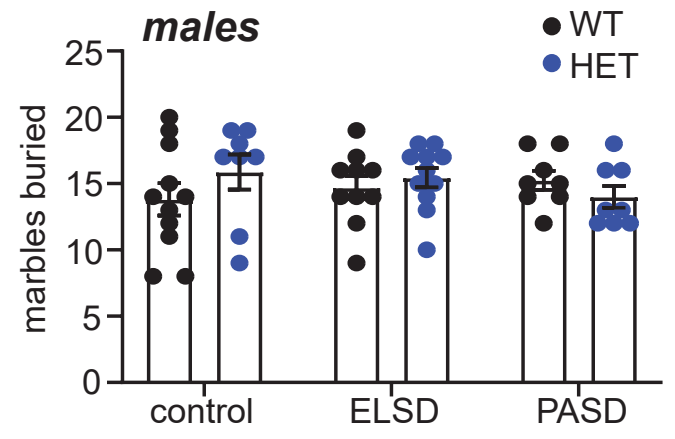

E olfaction test

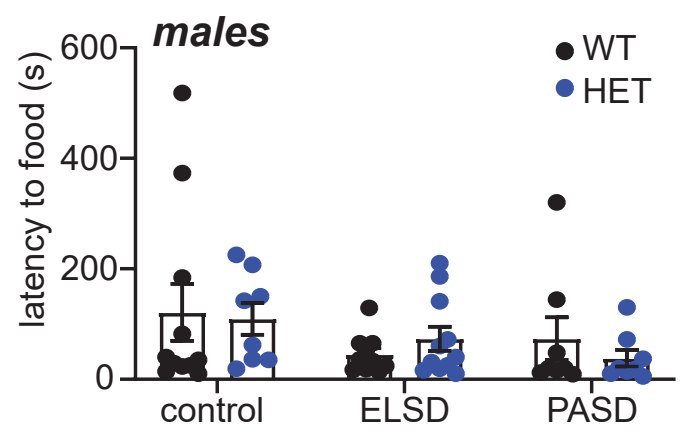

B
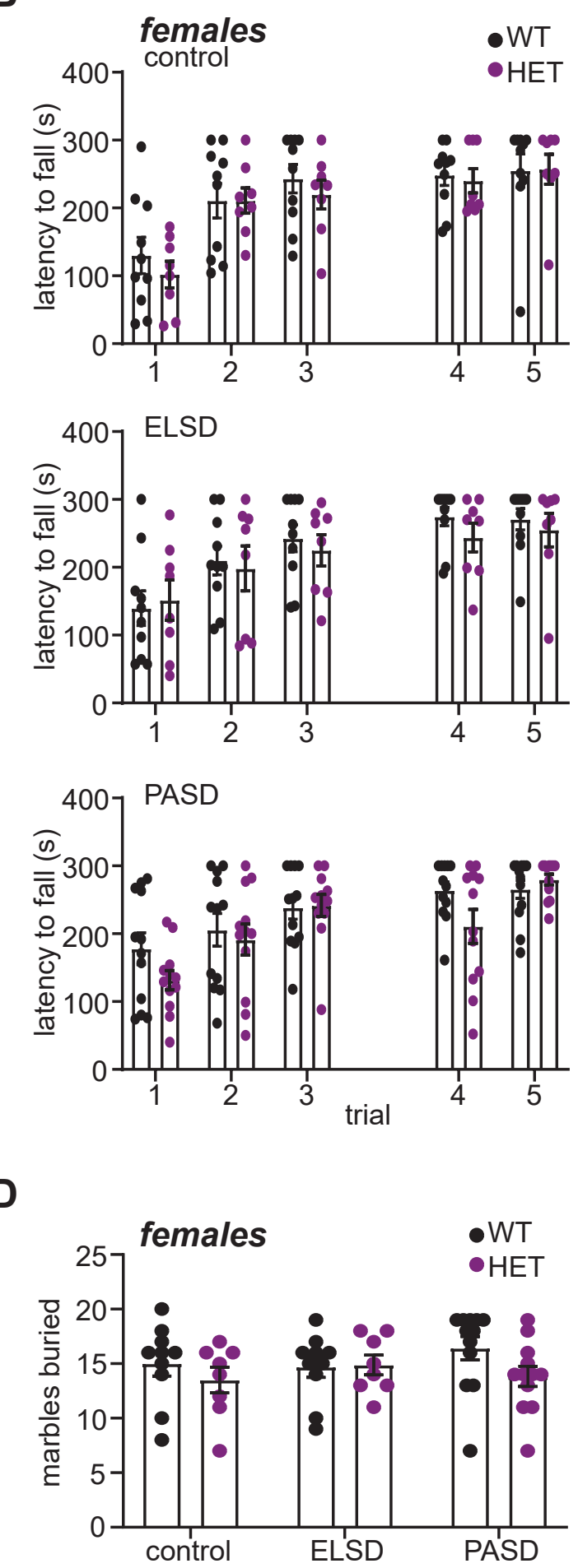

$\mathbf{F}$

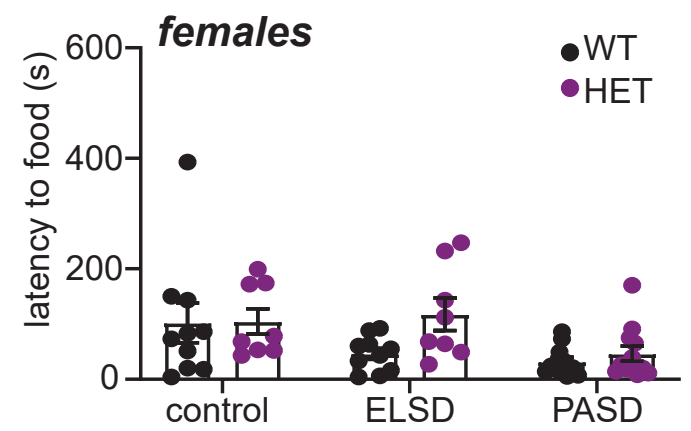


Figure 3 - figure supplement 2
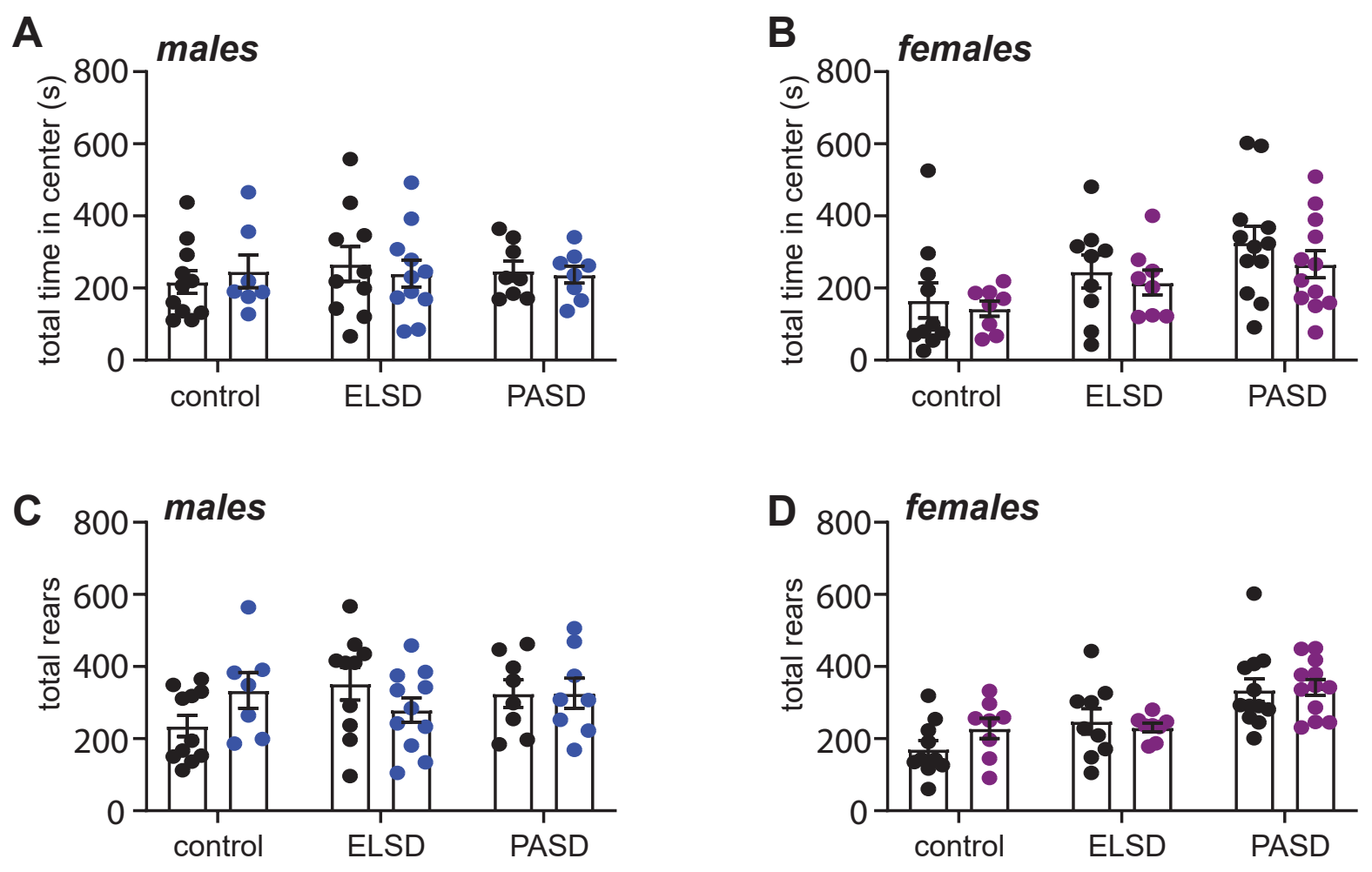